\title{
TUTORIA E MÚLTIPLAS ABORDAGENS EM OFICINA DE MODELAGEM E IMPRESSÃO 3D
}

ArthurR.Costa-arreico@gmail.com

Filipe A. Batista-filipe.aziz@gmail.com

Larissa P. C. Santos - larissapsanntos@gmail.com

Marcus J. A. Batista-marcus.jesse1@gmail.com

Dianne M.Viana-diannemv@unb.br

Jones Y. M. A. Silva-jonesyudi@unb.br

Faculdade de Tecnologia

Emilly M. B. Cornelio - emilly.mbcornelio@gmail.com

Faculdade do Gama, Campus do Gama

Fernanda R. Silva-fernandarose27@gmail.com

Faculdade de Educação

Carla M. C. C. Koike-ckoike@unb.br

Instituto de Ciências Exatas, Departamento de Ciência da Computação

Universidade de Brasília

Campus Darcy Ribeiro, L3 Norte

70910-900 - Brasília - DF

Resumo: $O$ desenvolvimento de habilidades e competências do século 21 passa por uma educação ativa, digital e flexível, centrada no estudante e na solução de problemas. Tal fato implica em mudança de paradigmas no que diz respeito à formação de monitores, tutores $e$ professores para atuar nas áreas STEM - ciência, tecnologia, engenharia e matemática -, uma vez que, estes foram educados em um processo, ainda em grande parte, tradicional, conteudista e compartimentado. O propósito deste artigo é descrever o procedimento usado no planejamento pedagógico de uma oficina para estudantes do ensino médio. Utilizamos como exemplo de aplicação uma oficina sobre modelagem e impressão 3D. Estratégias de sala de aula invertida, aprendizado autônomo, uso de aplicativos mobile e jogos são coadjuvantes do ensino-aprendizagem instrucional de modelagem e impressão 3D. A proposta de execução da oficina é testada em um grupo de estudantes de graduação como parte de uma capacitação para tutoria, em que estes assumem o papel de aprendizes do Ensino Médio a fim de obterem conhecimento suficiente para posteriormente atuarem como tutores e aplicarem a mesma oficina para estudantes do Ensino Médio. Reflexões acerca dos resultados da execução da oficina, eficácia do uso de metodologias ativas junto ao processo de ensino-aprendizagem e impressões a respeito das dificuldades encontradas pelos tutores ao assumirem o papel de estudantes do ensino médio são discutidas no âmbito de sua capacitação, além de utilizadas para identificar possíveis melhorias em oficinas posteriores.

Palavras-chave: Oficina pedagógica, STEM, Metodologias ativas. 


\section{INTRODUÇÃO}

Diante dos avanços e do entretenimento proporcionados pela tecnologia e a inevitável inserção de novas ferramentas em sala de aula, manter a atenção e aprendizagem dos alunos pelos métodos tradicionais se mostra cada vez mais difícil, sobretudo nas áreas de STEM, onde o conhecimento prático precisa ser especialmente consolidado. Em razão disso, o uso de metodologias tradicionais perde sua força diante das mudanças que o sistema educacional do século XXI passa, estimulando educadores a adotar novos métodos de ensino mais eficientes.

Nesse contexto, as metodologias de aprendizagem ativa se propõem a imbuir mais conhecimento de forma mais dinâmica e interativa, na forma de jogos, pesquisas, debates, entre outros. Entretanto, implementar um método de ensino tão diferente do que tanto os tutores quanto os alunos já estão acostumados se apresenta um grande desafio.

Segundo Prince e Felder (2006), as metodologias de ensino tradicionais que geralmente são aplicadas em cursos de engenharia acarretam em uma aprendizagem desestimulante e motivada apenas pelo desejo de não falhar em possíveis avaliações, pelo fato de não abordarem desde o início das aulas as aplicações específicas do conteúdo que será ministrado. Em contrapartida, as metodologias de aprendizagem ativa utilizam estudos de caso, observações específicas, experimentos e problemas para a partir disso ensinar teorias gerais, dando desde o início razões para que o estudante se mantenha ativo nos estudos.

À vista disso, convém utilizar as metodologias ativas a fim de melhorar a aprendizagem de estudantes em sala de aula. De acordo com Felder e Brent (2009), as metodologias de aprendizagem ativa se referem a qualquer atividade relacionada a disciplina estudada que não seja apenas assistir a aula de forma passiva. Seguindo nessa linha de raciocínio e citando Valente (2013), o estudante que vivencia uma aprendizagem ativa, resolve e discute problemas, desenvolve projetos, constrói protótipos, faz experimentos, utiliza métodos diferentes para aprender e, com isso, explora novas oportunidades para obtenção e construção de conhecimento. Por possibilitar o uso de novas ferramentas capazes de promover motivação e engajamento, as metodologias de aprendizagem ativa podem ser muito eficazes na consolidação de conhecimentos obtidos, proporcionando um aprendizado efetivo. Em face do que foi exposto, considera-se que o uso de um conjunto de estratégias de aprendizagem ativa pode trazer um resultado eficaz para o aprendiz.

No presente trabalho foram utilizadas metodologias ativas na produção e ensino de uma oficina de modelagem e impressão 3D. Uma delas foi a metodologia flipped classroom, traduzida como "sala de aula invertida". Nela, os professores se encarregam de elaborar pequenas atividades ou/e reunir vídeos, textos ou qualquer material para ser enviado aos estudantes a fim de que o aluno tenha um contato prévio com os assuntos a serem ministrados em sala de aula. O professor também pode consultar a atividade feita para identificar os assuntos de maior dificuldade dos alunos e utilizar mais tempo da aula para esclarecê-los (HERREID e SCHILLER, 2013).

Os tutores que ministraram as aulas também utilizaram metodologias ativas no processo de estudo das aulas, tendo em vista que não revisaram os conteúdos da oficina de forma passiva, mas ao planejarem aulas, tornaram-se protagonistas na busca por conhecimento a fim de resgatarem conteúdos já estudados e conseguir ensiná-los a outras pessoas. Durante o planejamento de uma aula o estudante que atua como tutor deve aprender ou revisar conteúdos, produzir materiais referentes a aula e esclarecer dúvidas de outros alunos, sendo esta última uma das tarefas mais desafiadoras, já que põe à prova os conhecimentos do tutor.

O propósito deste artigo é descrever o procedimento usado no planejamento pedagógico de uma oficina para estudantes do ensino médio, analisando-se as habilidades trabalhadas tanto no tutor quanto no aprendiz, bem como as dificuldades observadas neste processo. A 
oficina aqui apresentada faz parte de um conjunto de três oficinas sobre robótica aplicadas em três dias seguidos, sendo elas: I. Modelagem e Impressão 3D, II. Programação com Arduino e III. Integração de conhecimentos - Montagem e teste de um carrinho robótico. Este artigo relata a elaboração, execução e resultados da primeira oficina como parte de um procedimento de treinamento de tutores. Os tutores participam de projetos de extensão, elaborando e executando oficinas de STEM para estudantes do ensino médio em escolas públicas.

No procedimento para o treinamento dos tutores os estudantes de graduação desempenham três papéis: os estudantes responsáveis pela elaboração e realização das oficinas são os tutores; os estudantes que participam das oficinas e testam-nas são os aprendizes (estes replicarão as oficinas como atividades de projetos de extensão junto aos estudantes de ensino médio); há ainda o papel de observador, responsável por identificar possíveis problemas durante a execução dos testes .

\section{O PAPEL DO TUTOR DE STEM NO EMPREGO DA APRENDIZAGEM ATIVA}

Segundo Hernandez et al. (2014), a educação em STEM deve fazer uso do caráter interdisciplinar de matemática e ciência como forma de promover o desenvolvimento da compreensão dos alunos sobre engenharia e tecnologia. Pelo mesmo ângulo, Taajamaa et al. (2013) indicam que a formação de engenheiros deve desenvolver não só competências técnicas, ressaltando a importância do desenvolvimento de habilidades interdisciplinares e comportamentais - como cooperação e pensamento lateral - que permitirão a resolução de problemas complexos e reais.

Nesse sentido Tharayil et al. (2018), citando Freeman et al. (2014), Michael (2006), Prince (2004), Prince \& Felder (2007) e Lund e Stains (2015), mostram que a leitura tradicional dos métodos passivos de aprendizagem têm se provado menos eficazes do que as metodologias de aprendizagem ativa, que conduzem à melhora do aprendizado, à diminuição da evasão dos estudantes em programas de STEM e inúmeros outros resultados desejáveis, bem como o desenvolvimento do pensamento crítico e de habilidades de escrita e melhora da comunicação em sala de aula, conforme Bonwell e Eison (1991). Além disso, Topaz e Ziegelmeier (2015) e Bromley (2013) salientam que o método da sala de aula invertida é adaptável e capaz de aumentar o engajamento e a autonomia dos alunos na aula, além de aproximá-los dos professores.

Mesmo vantajoso, existem grandes dificuldades em deixar a metodologia passiva e conteudista à qual tanto os estudantes quanto os professores estão acostumados, bem como questões a respeito do papel do professor dentro desses métodos mais centrados no estudante. Uma análise de Tharayil et al. (2018) evidencia que, embora estudantes geralmente respondam positivamente ao uso das metodologias ativas, alguns mostram resistência, se mantendo indiferentes na realização das atividades, relutantes em responder perguntas do professor ou mesmo em fazer as perguntas em caso de dúvidas.

Os autores mostram que, para superar os impasses, é importante que o instrutor crie um ambiente interativo e confortável para que os aprendizes possam fazer perguntas e que não tenham medo de cometer erros na sala de aula. Isso pode ser feito através de pequenas mudanças no que é dito e em como é dito. Ressalta-se que os tutores de engenharia devem ter conhecimento dos componentes pessoais e afetivos essenciais para o sucesso da implementação das metodologias ativas.

Similarmente, Bromley (2013) cita Meyers e Jones (1993) ao enfatizar que a aprendizagem ativa exige que os tutores criem oportunidades para que os estudantes participem ativamente, discutindo e refletindo o conteúdo, ideias, questões e interesses de 
uma disciplina acadêmica. A autora também mostra que é possível adequar as metodologias ativas a cada forma de aprendizado.

Tharayil et al. (2018) também propõem andar pela sala enquanto os estudantes realizam alguma atividade, o que facilita a comunicação entre professor e aluno, tornando-a mais confortável, além de permitir a visualização das dificuldades e da participação de cada estudante. O trabalho também apontou a eficácia de se criar uma rotina e explicar o propósito do curso e das atividades. No caso dos alunos indiferentes durante as atividades, foi proposto que eles fossem chamados diretamente a participar, que os professores fizessem perguntas a respeito da atividade ou identificassem se havia alguma dificuldade. Outra forma de promover engajamento, é falar com os estudantes individualmente ou em grupos e fazer perguntas usando o silêncio como forma de incitar respostas.

Para McDonald (2016) é necessário que se invista no desenvolvimento profissional dos professores de STEM para a adoção de novas estratégias. Tharayil et al. (2018) lembram que se deve considerar que cada professor tem diferentes estilo e contexto educacional para a adoção de novas estratégias pedagógicas.

\section{PLANEJAMENTO DA OFICINA DE MODELAGEM E IMPRESSÃO 3D}

Um conjunto de três oficinas foi proposto para oportunizar uma experiência motivacional com o ensino-aprendizagem de robótica, envolvendo aspectos de modelagem, fabricação, montagem e teste de um carrinho de brinquedo para estudantes do ensino médio. As oficinas foram concebidas para serem executadas em 12 horas presenciais, divididas em três dias: Dia 1 - Modelagem e Impressão 3D; Dia 2 - Programação com Arduino e Dia 3 - Integração de Conhecimentos (montagem e teste do carrinho robótico).

Para tornar possível a realização das oficinas, o desenvolvimento do carrinho foi efetivado a partir de uma metodologia de projeto de engenharia com as seguintes fases: i) projeto conceitual, ii) projeto mecânico, iii) projeto eletrônico, iv) modelagem CAD; v) prototipagem; vi) testes. Dessa forma, foi construído o primeiro protótipo, avaliado e desenvolvidos mais dois protótipos do carrinho antes de se definir o modelo final, que consiste de 7 partes a serem fabricadas em PLA com tecnologia FDM (Full Deposition Modeling), a um custo aproximado de 4 horas de modelagem CAD e 10 horas de fabricação (Figura 1).

Figura 1 - Modelo final do carrinho com 135,0 mm de comprimento, 113,0 mm de largura e 37,5 mm de altura.
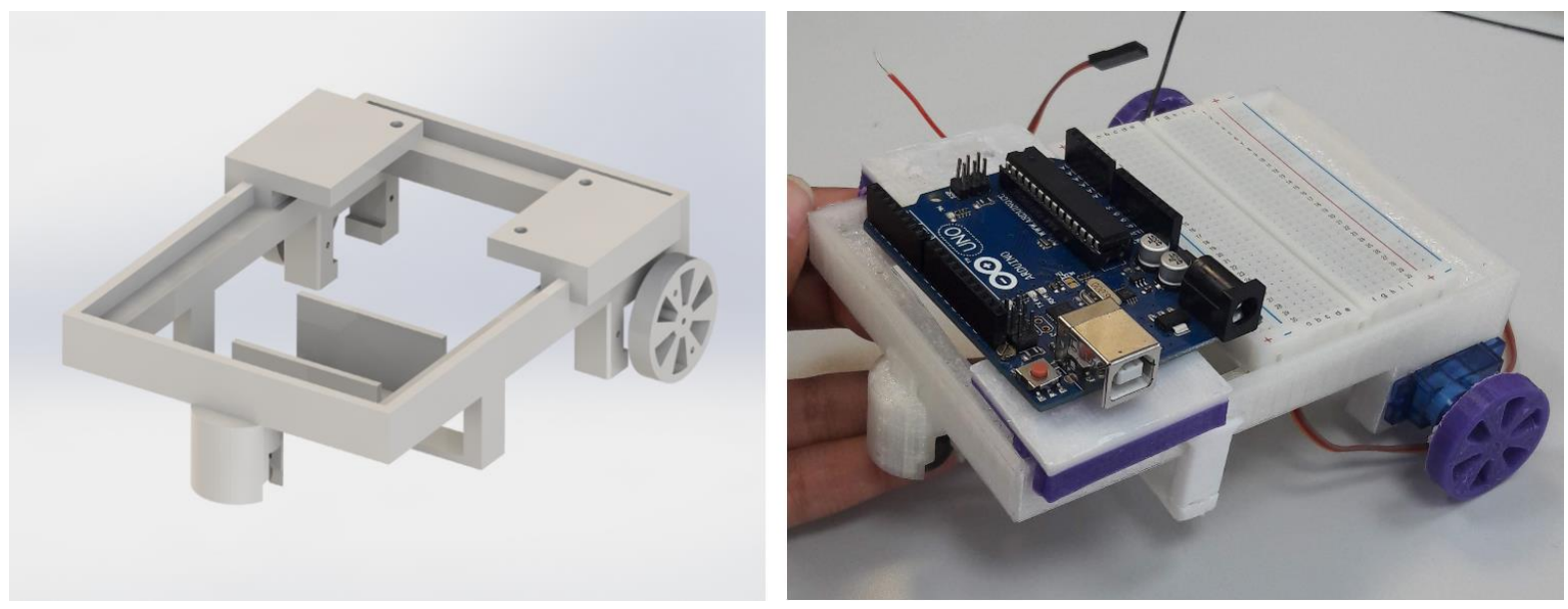

Fonte: Os autores. 
O projeto e prototipagem do carrinho, bem como o planejamento das oficinas foi realizado por uma equipe de 5 estudantes de cursos de engenharia e 1 estudante de curso de pedagogia sob a orientação de duas professoras de cursos de engenharia.

De uma maneira resumida, o planejamento da oficina, realizado após a prototipagem do modelo final do carrinho está apresentado no Quadro 1.

A oficina Modelagem e Impressão 3D foi pensada para introduzir também conceitos de torque e transmissão de potência no escopo do projeto do carrinho, não havendo a preocupação de se modelar e fabricar todas as partes do carrinho, mas a roda, como um elemento representativo.

A organização da oficina pode ser dividida em quatro etapas: estudo prévio com disponibilização de recursos para consulta; avaliação diagnóstica e revisão de conceitos (torque, velocidade angular e transmissão de potência mecânica); desenho do componente do carrinho no software Solidworks; manufatura aditiva.

Quadro 1 - Planejamento da oficina Modelagem e Impressão.

\begin{tabular}{|c|c|c|c|c|}
\hline \multirow{2}{*}{\multicolumn{2}{|c|}{ Etapas/Tempo }} & \multirow[t]{2}{*}{ Estratégia/ Recurso } & \multicolumn{2}{|c|}{ Habilidades trabalhadas } \\
\hline & & & Tutor & Aprendiz \\
\hline \multicolumn{2}{|c|}{$\begin{array}{l}\text { Desenvolvimento do roteiro } \\
1 \mathrm{~h}\end{array}$} & Descrição & $\begin{array}{l}\text { Interpretar e } \\
\text { planejar }\end{array}$ & Não se aplica \\
\hline \multicolumn{2}{|c|}{$\begin{array}{l}\text { Preparação dos materiais } \\
60 \mathrm{~h}\end{array}$} & $\begin{array}{l}\text { Hands on/ } \\
\text { equipamentos }\end{array}$ & $\begin{array}{l}\text { Criatividade, uso } \\
\text { de ferramentas }\end{array}$ & Não se aplica \\
\hline \multicolumn{2}{|c|}{$\begin{array}{l}\text { Avaliação do planejamento } \\
1 \mathrm{~h}\end{array}$} & Observação & $\begin{array}{l}\text { Pensamento } \\
\text { crítico }\end{array}$ & $\begin{array}{l}\text { Pensamento } \\
\text { crítico }\end{array}$ \\
\hline \multirow{3}{*}{$\begin{array}{l}\mathrm{o} \\
\mathrm{f} \\
\mathrm{i}\end{array}$} & $\begin{array}{l}\text { Estudo prévio } \\
1 / 2 \mathrm{~h}\end{array}$ & Aula invertida / celular & $\begin{array}{l}\text { Investigação, } \\
\text { criatividade }\end{array}$ & Leitura \\
\hline & $\begin{array}{l}\text { Revisão de conceitos } \\
1 / 2 \mathrm{~h}\end{array}$ & $\begin{array}{l}\text { Avaliação diagnóstica / } \\
\text { questionário } \\
\text { Exposição interativa }\end{array}$ & $\begin{array}{l}\text { Pensamento } \\
\text { crítico, empatia, } \\
\text { comunicação }\end{array}$ & $\begin{array}{l}\text { Consolidação de } \\
\text { conceitos }\end{array}$ \\
\hline & $\begin{array}{l}\text { Modelo CAD } \\
1 \mathrm{e}^{1 / 2} \mathrm{~h}\end{array}$ & $\begin{array}{l}\text { Instrução passo a passo } \\
\text { / computador }\end{array}$ & $\begin{array}{l}\text { Planejamento, } \\
\text { visão espacial }\end{array}$ & $\begin{array}{l}\text { Representação } \\
\text { gráfica, visão } \\
\text { espacial }\end{array}$ \\
\hline $\begin{array}{l}\mathrm{n} \\
\mathrm{a}\end{array}$ & $\begin{array}{l}\text { Manufatura Aditiva } \\
1 \mathrm{~h}\end{array}$ & Impressora 3D & $\begin{array}{l}\text { Comunicação, } \\
\text { uso de } \\
\text { ferramentas }\end{array}$ & $\begin{array}{l}\text { Representação } \\
\text { física, visão } \\
\text { espacial }\end{array}$ \\
\hline
\end{tabular}

Fonte: Os autores. 
Optou-se por apresentar a modelagem da roda do carrinho, por questões de simplicidade e limitação de tempo, por ser uma peça pequena de 29,4 mm de diâmetro e $6 \mathrm{~mm}$ de espessura e sua impressão ser concluída em aproximadamente 25 minutos. A atividade foi planejada para ser guiada por um dos tutores, o qual apresentava os passos do desenho que seriam replicados pelos aprendizes. Além disso, para engajar os aprendizes e tornar mais interessante a atividade, esta foi planejada na forma dialogada. Desse modo, durante a apresentação das etapas do desenho o tutor apresenta ideias e questionamentos e debate com os aprendizes, propiciando um diálogo.

Por fim, a apresentação sobre manufatura aditiva foi planejada de modo a propiciar uma reflexão sobre as vantagens e desvantagens desta em relação aos processos tradicionais de fabricação.

\section{EXECUÇÃO DA OFICINA}

Participaram da execução da oficina, 10 estudantes de graduação de cursos de engenharia e da área de humanidades. Destes, 4 estudantes participaram do planejamento da oficina sob orientação de professores e atuaram como tutores, 1 estudante de pedagogia participou como observadora e os demais participaram como aprendizes. Os estudantes de graduação que participaram como aprendizes seriam responsáveis por replicar as oficinas para estudantes de ensino médio.

Utilizou-se a estratégia flipped classroom para a introdução de conceitos e consolidação de conhecimentos sobre máquinas simples e conceitos físicos como torque e potência. A parte referente aos estudos prévios começou no dia anterior ao da realização da oficina, quando foi enviado aos aprendizes vídeos, textos e notícias sobre conceitos físicos que seriam trabalhados em sala de aula.

A avaliação diagnóstica foi realizada por meio de um quiz, produzido na plataforma Kahoot! no início da oficina e abordou questões de múltipla escolha sobre torque, potência e engrenagens. Cada questão foi lida em voz alta pelo tutor responsável e, após leitura, no tempo disponível para que todos pudessem responder cada pergunta, os alunos puderam discutir possíveis respostas com seus colegas. Este mesmo quiz apresenta uma colocação com pontuações após término do teste, o que empolgou os alunos.

Após término do quiz, foram revisados conceitos relacionados a máquinas simples e apresentados exemplos de alavanca, roldana e polia, dando enfoque para engrenagens, em particular a engrenagem cilíndrica de dentes retos apresentando e discutindo conceitos de redução de velocidade em um par de engrenagens, bem como, conceitos de torque e potência. Durante a explicação teórica usou-se exemplos cotidianos presentes na vida dos alunos para que os conceitos fossem melhor assimilados, conforme recomenda Srinath (2014).

Para a modelagem CAD da roda do carrinho foi utilizado o Solidworks (Figura 2). Nenhum dos alunos presentes havia usado anteriormente este software, todavia, durante toda a parte prática os problemas que apareceram foram rapidamente resolvidos com a ajuda de tutores. Com a tela do software projetada para os alunos, o tutor responsável desenhou a roda pausadamente, explicando as funções utilizadas para isso, seguido dos alunos que reproduziam cada um dos passos feitos pelo tutor (Figura 3). Os termos técnicos utilizados foram também esclarecidos.

O tutor, utilizando a biblioteca Toolbox no Solidworks, mostrou também como desenhar uma engrenagem cilíndrica de dentes retos.

Por fim, na última etapa da oficina houve uma apresentação sobre os diferentes tipos de manufatura e as principais tecnologias de impressão 3D. A visita ao Laboratório Aberto de 
Brasília foi feita na hora final da oficina e os aprendizes puderam ver sua modelagem CAD ser enviada para a impressora e por fim ser impressa.

Figura 2 - Projeto da rodinha com 29,4 $\mathrm{mm}$ de diâmetro e $6 \mathrm{~mm}$ de espessura.

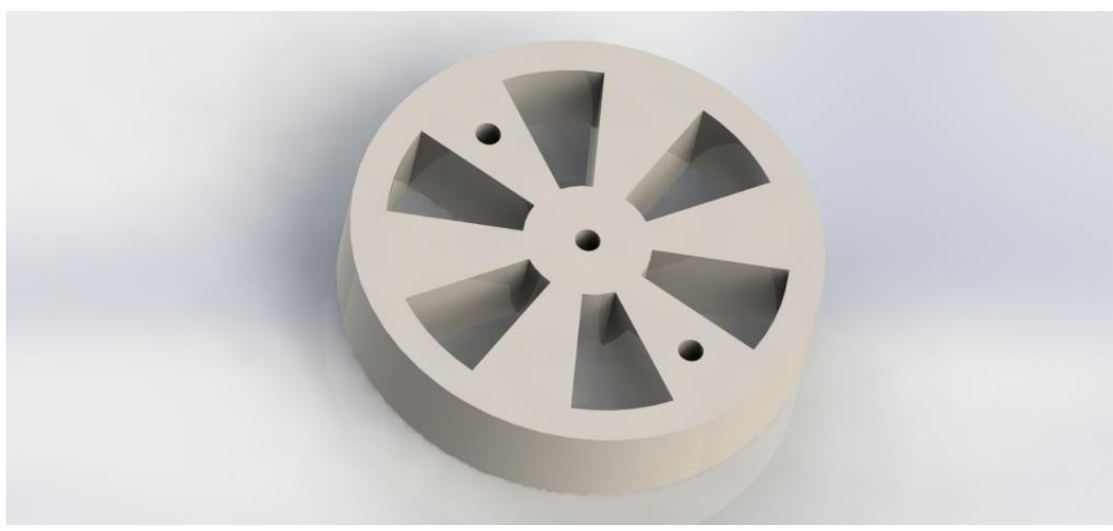

Fonte: Os Autores.

Figura 3 - Alunos em sala de aula fazendo a modelagem da peça.

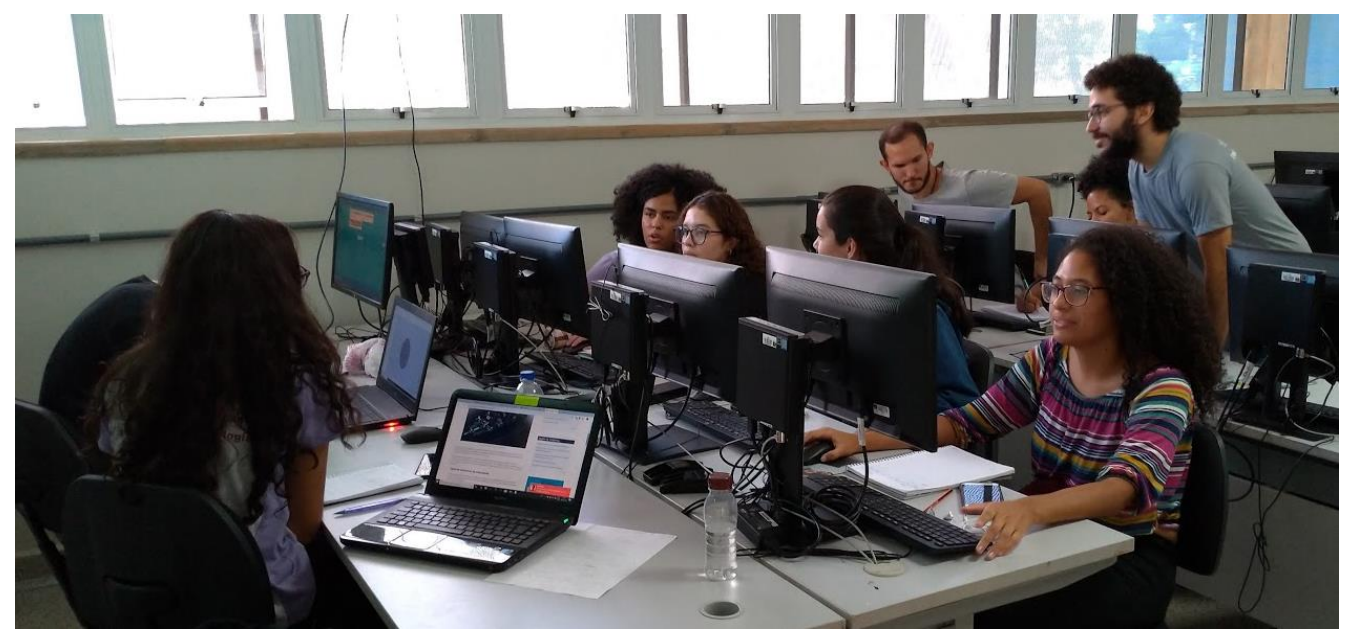

Fonte: Os autores.

\section{RESULTADOS}

Para analisar o uso das metodologias de aprendizagem ativa e a tutoria na oficina, três tutores e três aprendizes responderam questionários via Google Forms.

Os estudantes com o papel de tutores, responderam de forma favorável quanto à avaliação do uso das metodologias de aprendizagem ativa no processo de ensinoaprendizagem, mas afirmaram ter tido dificuldades em explicar de forma didática.

No planejamento da oficina houve dúvidas em como conectar os temas e explicar de forma didática. Aspectos que também apareceram no que poderia ser melhorado: as explicações dos conceitos físicos e a abordagem dos temas. Além disso, foram sugeridos o uso de explicações simplificadas e uma melhor gestão do tempo.

No que diz respeito a criar oportunidades para estimular o pensamento crítico e a autonomia dos estudantes, a maioria disse que o fez apenas às vezes.

Também foi ressaltada a heterogeneidade da participação dos aprendizes nas oficinas: alguns participaram ativamente das atividades, enquanto outros se mostraram indiferentes. 
Em relação à questão sobre o que a realização da oficina acrescentou no aprendizado do tutor em tal condição, foi dito que permitiu-se o aprendizado de algo antes mesmo de estudálo dentro do curso, a percepção da necessidade de melhorar a própria didática e formas de realizar oficinas melhores no futuro, tendo-se portanto, como autoavaliação em uma escala de 0 a 10 , resultados de 6 a 8 .

Enquanto aprendizes, durante a capacitação, os estudantes apontaram melhoras a serem feitas na didática, considerando o público da oficina, sugerindo uso de mais exemplos para aproximar o conteúdo do cotidiano dos estudantes do ensino médio, bem como mais tempo destinado a cada atividade. Respostas idênticas às dadas sob o ponto de vista dos tutores na oficina.

Durante o treinamento houve dificuldade no uso do Solidworks para modelagem das peças, por falta de prática ou familiaridade, mas todos conseguiram utilizá-lo.

Tendo isso em vista, o uso das metodologias de aprendizagem ativa também foi aprovado com unanimidade. Todos concordaram que a prática leva a um melhor entendimento da teoria e todos consultaram antes da aula o material fornecido previamente e o consideraram importante tanto para a compreensão da aula quanto para a realização da oficina.

Também houve consonância em conseguir relacionar a explicação dos conceitos físicos às atividades das oficinas, mas um dos aprendizes se mostrou insatisfeito com o entendimento da teoria. Da mesma forma, dois dos três estudantes se mostraram seguros sobre visualizar aplicações cotidianas dos assuntos abordados, enquanto o restante não sabia.

Sobre os conteúdos mais importantes entre os apresentados na aula, as opiniões foram diversas. Um dos aprendizes considerou que todos foram importantes e contribuíram para um melhor entendimento dos mecanismos usados no cotidiano, outro apontou o uso do Solidworks, o último julgou serem os conceitos físicos apresentados, mencionando também a modelagem 3D. Segundo eles, as oficinas proporcionaram em relação ao aprendizado enquanto tutor: novas ideias e estímulo a buscar e oportunizar a construção de conhecimento, melhora da didática e a visualização da importância do trabalho em equipe para o funcionamento das oficinas

\section{CONSIDERAÇÕES FINAIS}

O procedimento usado no planejamento pedagógico da oficina Modelagem e Impressão 3D foi descrito, destacando-se aspectos do projeto do carrinho e habilidades trabalhadas no tutor e no aprendiz. Segundo os resultados observados, os objetivos propostos foram alcançados, propiciando aos tutores uma experiência de planejamento, elaboração e execução da oficina, e aos aprendizes, oportunidades de aprendizagem quanto à modelagem 3D e manufatura aditiva, além de vivenciarem uma situação que o estudante de ensino médio vivenciará também, quando a oficina for replicada para eles. A realização da oficina também ajudou na identificação de possíveis melhorias a serem implementadas, no que diz respeito à abordagem dos conceitos e gestão do tempo. As metodologias ativas se mostraram eficazes no processo de aprendizagem tanto para os tutores, quanto para os aprendizes, como evidenciado nas respostas aos questionários. Ainda assim, foram observadas dificuldades em engajar os alunos em algumas atividades. Os resultados e a comparação destes com o planejamento mostram que é necessário adequar as atividades para um tempo menor e incentivar mais a participação dos aprendizes.

\section{Agradecimentos}

À FAP-DF pelo apoio financeiro concedido por meio do Edital 03/2018. 
"Os desafios para formar hoje o engenheiro do amanhã"

\section{REFERÊNCIAS}

BONWELL, Charles; EISON, James. Active Learning: Creating Excitement in the Classroom. Washington, DC: ASHE-ERIC Higher Education Report 1, 1991.

BROMLEY, Pam. Active learning strategies for diverse learning styles: Simulations are only one method. PS: Political Science \& Politics, v. 46, n. 4, p. 818-822, 2013.

FELDER, Richard M.; BRENT, Rebecca. Active Learning: An Introduction. ASQ HIGHER EDUCATION BRIEF, v. 2, p. 1-5, 2009.

FREEMAN, Scott et al. Active learning increases student performance in science, engineering, and mathematics. Proceedings of the National Academy of Sciences, v. 111, n. 23, p. 8410-8415, 2014.

HERREID, Clyde Freeman; SCHILLER, Nancy A. Case studies and the flipped classroom. Journal of College Science Teaching, v. 42, n. 5, p. 62-66, 2013.

HERNANDEZ, Paul R., et al. Connecting the STEM dots: Measuring the effect of an integrated engineering design intervention. International Journal of Technology and Design Education, 2014.

LUND, Travis J.; STAINS, Marilyne. The importance of context: an exploration of factors influencing the adoption of student-centered teaching among chemistry, biology, and physics faculty. International Journal of STEM Education, v. 2, n. 1, p. 13, 2015.

MCDONALD, Christine. STEM Education: A review of the contribution of the disciplines of science, technology, engineering and mathematics. Science Education International, v. 27 , n. 4, p. 530-569, 2016.

MEYERS, Chet; JONES, Thomas B. Promoting Active Learning. Strategies for the College Classroom. Jossey-Bass Inc., Publishers, 350 Sansome Street, San Francisco, CA 94104, 1993.

MICHAEL, Joel. Where's the evidence that active learning works? American Physiological Society, v. 30, p. 159-167, 2006.

PRINCE, Michael. Does active learning work? A review of the research. Journal of Engineering Education, v. 93, p. 223-231, 2004.

PRINCE, Michael J.; FELDER, Richard M. Inductive teaching and learning methods: Definitions, comparisons, and research bases. Journal of engineering education, v. 95, n. 2, p. 123-138, 2006.

PRINCE, Michael; FELDER, Richard. The many faces of inductive teaching and learning. Journal of college science teaching, v. 36, n. 5, p. 14, 2007.

SRINATH, Adusumilli. Active Learning Strategies: An illustrative approach to bring out better learning outcomes from Science, Technology, Engineering and Mathematics (STEM) 
students. INTERNATIONAL JOURNAL OF EMERGING TECHNOLOGIES IN

LEARNING, v. 9, n. 9, p. 21-25, 2014.

TAAJAMAA, Ville et al. Dancing with Ambiguity Design thinking in interdisciplinary engineering education. In: 2013 IEEE Tsinghua International Design Management Symposium. IEEE, p. 353-360, 2013.

THARAYIL, Sneha et al. Strategies to mitigate student resistance to active learning. IJ STEM, v. 5, n. 7, p. 1-16, 2018.

TOPAZ, Chad; ZIEGELMEIER, Lori. Flipped Calculus: A Study of Student Performance and Perceptions. PRIMUS, v. 25, n. 9-10, p. 847-860, 2015.

VALENTE, José Armando. Aprendizagem Ativa no Ensino Superior: a proposta da sala de aula invertida. p. 1-4, 2013.

\title{
TEACHING AND MULTIPLE APPROACHES IN 3D MODELING AND PRINTING WORKSHOP
}

\begin{abstract}
The development of skills and competences of the 21st century passes through an active, digital and flexible education, focused on the student and on problem solving. This fact implies a shift in paradigms regarding the training of monitors, tutors and teachers to work in the STEM areas - science, technology, engineering and mathematics -, since they were educated in a process, still largely, traditional, conteudist and compartmentalized. The purpose of this article is to describe the procedure used in the pedagogical planning of a workshop for high school students. We use as an example of application a 3D modeling and printing workshop. Flipped classroom strategies, autonomous learning, use of mobile applications and games are coadjuvants of the instructional teaching-learning of $3 D$ modeling and printing. The proposal of the workshop is tested in a group of undergraduate students as part of a tutoring training, in which they take on the role of high school apprentices in order to obtain enough knowledge to later act as tutors and apply the same workshop to high school students. Reflections on the results of the workshop execution test, the effectiveness of the use of active methodologies along with the teaching-learning process, and impressions regarding the difficulties encountered by tutors in assuming the role of high school students are discussed in the context of their training and used to identify possible improvements in later workshops.
\end{abstract}

Keywords: Pedagogical workshop, STEM, Active methodologies. 